\title{
Carcinoma colorrectal en niños y adolescentes
} Colorectal carcinoma in children and adolescents

\author{
Dra. Marina D. Matheya, Dra. Carla L. Pennella y Dr. Pedro Zubizarreta ${ }^{a}$
}

\section{RESUMEN}

Si bien el carcinoma colorrectal (CCR) es la tercera enfermedad oncológica más frecuente en adultos, solo el 1-4\% ocurre en menores de 25-30 años. Su presentación suele confundirse con otras patologías, lo cual lleva a significativas demoras diagnósticas. Dada su baja incidencia, pocos pediatras se enfrentarán con algún caso a lo largo de su carrera. Sin embargo, existen en la niñez múltiples síndromes hereditarios que predisponen al CCR.

El objetivo de esta revisión es brindar una actualización sobrelos síndromes predisponentes alCCR. Se revisarán las indicaciones de tamizaje, dado que el diagnóstico precoz en estadios localizados es el principal factor pronóstico. Además, se fortalecerán las herramientas de consejería genética para el paciente y su familia. A su vez, se discutirán las manifestaciones clínicas e histológicas y los factores pronósticos propios del CCR en la población pediátrica. Si bien las guías de tratamiento se extrapolan de la experiencia en adultos, se resumirán los lineamientos terapéuticos.

Palabras clave: neoplasias colorrectales, niño, adolescente.

http: / / dx.doi.org/10.5546/ aap.2021.e487

Texto completo en inglés:

http: / / dx.doi.org/10.5546/ aap.2021.eng.e487

Cómo citar: Mathey MD, Pennella CL, Zubizarreta P. Carcinoma colorrectal en niños y adolescentes. Arch Argent Pediatr 2021;119(5):e487-e498.

a. Servicio de Hematología y Oncología, Hospital de Pediatría S.A.M.I.C. "Prof. Dr. Juan P. Garrahan", Ciudad Autónoma de Buenos Aires, Argentina.

Correspondencia: Dra. Marina Mathey: mmathey.mm@gmail. com

Financiamiento:

Ninguno.

Conflicto de intereses: Ninguno que declarar.

Recibido: 23-12-2020 Aceptado: 22-4-2021
La tasa anual de incidencia según los datos del Surveillance, Epidemiology and End Results (SEER) es de 0,12/1 000000 en la población entre 0 y 14 años, y se incrementa a 1,78/1 000000 entre los 15 y 19 años (Figura 1). 2,3 Sin embargo, es la enfermedad primaria maligna gastrointestinal más común, luego de los tumores hepáticos. ${ }^{4}$

\section{CARCINOGÉNESIS Y SÍNDROMES HEREDITARIOS PREDISPONENTES}

El CCR surge de la combinación de tres mecanismos de carcinogénesis: ${ }^{5}$

- Inestabilidad cromosómica: la mutación inactivadora del gen supresor de tumores adenomatous polyposis $(A P C)$ es el evento inicial que conlleva la desregulación en la homeostasis de la E-cadherina, con activación de protooncogenes (c-Myc y KRAS) e inactivación de genes supresores de tumores (p53). Este mecanismo está presente en el 65-70 \% de los tumores esporádicos y en la poliposis adenomatosa familiar. 5,6

- Inestabilidad de microsatélites (MSI, por su sigla en inglés): se debe a la mutación germinal de un alelo de los genes de apareamiento erróneo $(M M R$, por su sigla en inglés), seguida por la inactivación somática del otro. Estas mutaciones implican cambios de longitud por inserciones o deleciones de nucleótidos en secuencias repetidas e inestables del ADN, denominadas microsatélites. Para su estudio se utilizan 5 microsatélites marcadores estándares de referencia. Se denomina MSI de alta frecuencia (MSI-H, por su sigla en inglés) cuando dos o más 
de estos marcadores muestran cambios en su longitud, y es característica del síndrome de Lynch. ${ }^{5}$

- Fenotipo de islas de metilación CpG: está dado por la hipermetilación y el silenciamiento de genes supresores de tumores (MGMT y MLH1). Se asocia a mutaciones del gen BRAF y es exclusivo del $15 \%$ de los CCR esporádicos donde el silenciamiento transcripcional secundario a la metilación aberrante del gen MMR hMLH1 genera MSI-H. ${ }^{5}$

En pediatría, el 10-30 \% de los casos tienen historia familiar de CCR, todos asociados a síndromes genéticos predisponentes. ${ }^{1,7}$ Se pueden ordenar en tres grupos:

\section{- CCR asociado a poliposis:}

- Poliposis adenomatosa familiar (FAP).

- Síndrome de poliposis asociado a MUTYH (MAP).

- Síndrome de Peutz-Jeghers (SPJ).

- Síndrome de poliposis juvenil (PJS).

- Síndrome de poliposis hiperplásica juvenil.

- CCR hereditario no asociado a poliposis:

- Síndrome de Lynch o carcinoma colorrectal hereditario no polipósico (HNPCC).

- Deficiencia constitucional de los genes MMR (CMMR-D).
- Adenocarcinoma resultado de transformación maligna de la enfermedad inflamatoria intestinal

Es fundamental, para la pesquisa, una historia clínica completa con detallados antecedentes familiares que incluyan la identificación de todo tipo de cáncer, la edad de inicio, las características fenotípicas que puedan estar relacionadas y la documentación de hallazgos histopatológicos. ${ }^{8}$ En la Tabla 1 se resumen los criterios diagnósticos e indicaciones de tamizaje de los síndromes predisponentes más frecuentes.

\section{A. Poliposis adenomatosa familiar (FAP)}

Su incidencia es de $1 / 7000$ individuos. ${ }^{9}$ De herencia autosómica dominante, es causada por la mutación inactivadora del gen supresor de tumores APC. El 15-20 \% de los casos son de novo, por mutaciones adquiridas en dicho gen. - $^{9}$

Se caracteriza clínicamente por la presencia de cientos de adenomas colónicos en la preadolescencia con degeneración maligna a los 40-50 años. Presentan, además, adenomas periampulares o ampulares duodenales. ${ }^{11,12}$

Dada la alta penetrancia, los pacientes tienen un riesgo del $100 \%$ de desarrollar CCR a edades tempranas y un riesgo acumulativo de adenocarcinoma duodenal del $10 \%$ a los 60

FIGURA 1. Histograma que muestra la distribución por edad en (A) 550622 pacientes con CCR diagnosticados entre 1973 y 2005, y en (B) 159 niños y adolescentes diagnosticados durante el mismo perído

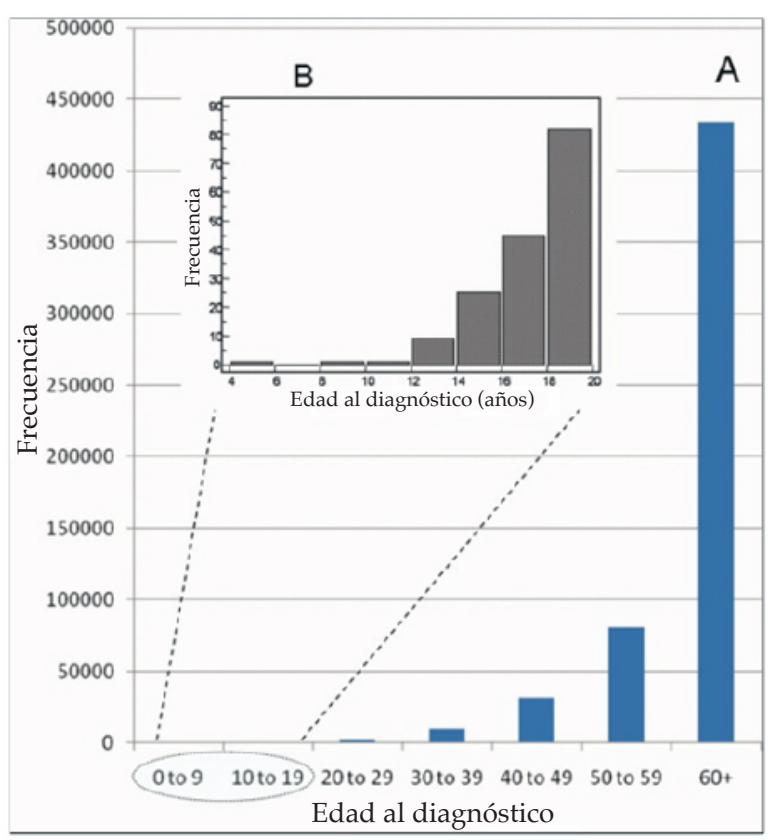

Adaptado de Sultan I, et al. ${ }^{3}$ 
años. ${ }^{11,13}$ La FAP se asocia con menos frecuencia a otras enfermedades malignas extracolónicas, como el carcinoma tiroideo y el hepatoblastoma. Como manifestaciones clínicas no malignas extraintestinales, se describen la hipertrofia congénita del epitelio pigmentario de la retina (CHRPE), la demora en la erupción dentaria, el osteoma mandibular y múltiples tumores fibroides y / o desmoides. ${ }^{10-12}$

Existe una forma de presentación atenuada con menor cantidad de pólipos (20-100 adenomas, sobre todo en el colon derecho) y aparición de CCR a edades más tardías, denominada poliposis adenomatosa familiar atenuada (AFAP, por su sigla en inglés). ${ }^{9,11}$

Dentro de la población pediátrica con CCR, el $10 \%$ se asocia a mutaciones del gen APC. Se presentan en forma localizada, en su mayoría en colon sigmoide o recto. La sobrevida global (SG) a 5 años es de $59 \%( \pm 12)$, significativamente mejor que la del resto del grupo pediátrico $(p=0,085){ }^{3}$
Los pacientes con mutaciones del gen APC y los familiares de primer grado requieren tamizaje con videoendoscopia digestiva alta (VEDA), videocolonoscopia (VCC) y cápsula endoscópica desde los 10-14 años. Se recomienda la colectomía total entre los 15 y los 18 años, incluso para los pacientes asintomáticos, a fin de evitar el desarrollo de CCR. ${ }^{11-16}$ Luego de la colectomía se debe continuar con controles endoscópicos anuales dado el riesgo de desarrollar manifestaciones extracolónicas. En los pacientes con AFAP, el estudio endoscópico se indica desde los 18-20 años. La colectomía profiláctica no estaría indicada. ${ }^{16}$

Dado el patrón de herencia, el $50 \%$ de los familiares de primer grado presentarán la misma alteración. ${ }^{10}$

\section{B. Síndrome de poliposis asociado a MUTYH (MAP)}

De herencia autosómica recesiva, es causado por la mutación germinal bialélica del gen

TABla 1. Sindromes hereditarios predisponentes, criterios diagnósticos y recomendaciones de tamizaje

\begin{tabular}{|c|c|c|}
\hline $\begin{array}{l}\text { Síndrome hereditario } \\
\text { (riesgo de CCR) }\end{array}$ & Criterios diagnósticos clínicos & Tamizaje y profilaxis \\
\hline $\begin{array}{l}\text { FAP } \\
(100 \%)\end{array}$ & $\begin{array}{l}\text { Ante ausencia de diagnóstico molecular, } \\
\text { sospechar: } \\
\text { - AP si }>100 \text { pólipos adenomatosos en } \\
\text { la } 2 \text {. }^{\text {da }}-3 \text {.ra década de vida. }^{\text {- }} \text { AFAP si }<100 \text { pólipos adenomatosos } \\
\text { de inicio entre } 4 .^{\text {ta }}-5 \text { ta }^{\text {ta }} \text { década de vida. }\end{array}$ & $\begin{array}{l}\text { - FAP: familiares de } 1 .^{\text {er }} \text { grado: } \\
\text { VEDA y VCC desde los 10-14 años. } \\
\text { Colectomía total entre los 15-18 años. } \\
\text { - AFAP: VEDA y VCC desde los 18-20 años. } \\
\text { Colectomía profiláctica: } \\
\text { no estaría indicada. }\end{array}$ \\
\hline $\begin{array}{l}\text { PJS } \\
(39 \%)\end{array}$ & $\begin{array}{l}\text { Uno de: } \\
\text { - } \quad>2 \text { pólipos hamartomatosos de tipo PJS. } \\
\text { - Cualquier cantidad de pólipos hamartomatosos } \\
\text { de tipo PJS e historia familiar de PJS. } \\
\text { - Alteraciones pigmentarias características e } \\
\text { historia familiar de PJS. } \\
\text { - Cualquier cantidad de pólipos hamartomatosos } \\
\text { tipo-PJS con alteraciones pigmentarias } \\
\text { características. }\end{array}$ & $\begin{array}{l}\text { VEDA, VCC y cápsula endoscópica } \\
\text { desde los } 8 \text { años: } \\
\text { - Si hay hallazgos positivos, } \\
\text { repetir cada } 3 \text { años. } \\
\text { - Si es normal, controles desde } \\
\text { los } 18 \text { años cada } 3 \text { años. } \\
\text { Desde los } 25-30 \text { años: mamografía, } \\
\text { PAP y controles ginecológicos periódicos; } \\
\text { en los varones, control clínico y } \\
\text { ecográfico testicular. }\end{array}$ \\
\hline $\begin{array}{l}\text { JPS } \\
(38 \%)\end{array}$ & $\begin{array}{l}\text { Al menos uno de: } \\
\text { - } \quad>5 \text { pólipos juveniles del colorrecto. } \\
\text { - } \quad \text { Múltiples pólipos juveniles en el tracto digestivo. } \\
\text { - } \quad \text { Cualquier cantidad de pólipos juveniles e } \\
\quad \text { historia familiar de pólipos juveniles. }\end{array}$ & $\begin{array}{l}\text { VEDA y VCC desde los } 15 \text { años cada } 3 \text { años. } \\
\text { Colectomía profiláctica: } \\
\text { poliposis no manejada por endoscopia, } \\
\text { sangrado o diarrea grave, pólipos juveniles } \\
\text { con displasia e historia familiar de CCR. }\end{array}$ \\
\hline $\begin{array}{l}\text { SL } \\
(80 \%)\end{array}$ & $\begin{array}{l}\text { Criterios de Ámsterdam II } \\
\text { - } \quad>3 \text { familiares con cáncer asociado a HNPCC. } \\
\text { Más todos los siguientes: } \\
\text { - } \quad \text { Entre ellos, un familiar de primer grado. } \\
\text { - } \quad \text { Dos generaciones sucesivas afectadas. } \\
\text { - } \quad \text { En uno de ellos, CCR antes de los } 50 \text { años. } \\
\text { - } \quad \text { Exclusión de FAP. }\end{array}$ & $\begin{array}{l}\text { VCC a partir de los 18-20 años, } \\
\text { cada } 1-2 \text { años. }\end{array}$ \\
\hline
\end{tabular}

CCR: carcinoma colorrectal. FAP: poliposis adenomatosa familiar. AFAP: poliposis adenomatosa familiar atenuada. PJS: síndrome de Peutz-Jeghers. JPS: Síndrome de poliposis juvenil. SL: síndrome de Lynch. VEDA,

VCC: videoendoscopia digestiva alta, videocolonoscopia. HNPCC: carcinoma colorrectal hereditario no polipósico. 
MUTYH, que codifica para un sistema de reparación del ADN.

Se caracteriza por un fenotipo leve de poliposis $(<100$ adenomas colorrectales al diagnóstico) y riesgo aumentado (93 veces) de CCR. Con presentación tardía, la edad media de diagnóstico de poliposis es en la adultez. El $58 \%$ de los pacientes presentarán CCR. ${ }^{12,14,16,17}$

En los pacientes con más de 100 adenomas colónicos se deben investigar mutaciones del gen $A P C$. De dar resultado negativo, se debe continuar con el estudio del gen MUTYH. Si no se detectan estas mutaciones, se recomienda continuar con el estudio de los genes POLE y POLD1. ${ }^{16}$

Se recomienda realizar VCC desde los 18-20 años, cada 2 años. ${ }^{9,12,14,17}$

\section{Síndrome de Peutz-Jeghers (PJS)}

La incidencia es de 1/250 000 individuos. De herencia autosómica dominante, es causado por la mutación germinal del gen supresor de tumores STK11/LKB1. El $45 \%$ no tiene historia familiar. ${ }^{16,18}$

Se caracteriza fenotípicamente por hiperpigmentaciones periorales y en los dedos, poliposis hamartomatosas en el tracto gastrointestinal, tumores en el intestino delgado y el páncreas, CCR y tumor de los cordones sexuales. Los pacientes con PJS tienen un riesgo relativo del 15,2 \% de desarrollar algún tipo de cáncer y del $39 \%$ de desarrollar CCR a cualquier edad. ${ }^{12,19}$

Se recomienda tamizaje con VEDA, VCC y cápsula endoscópica a los 8 años. Ante hallazgos positivos, repetir cada 3 años; si no, continuar con controles desde los 18 años cada 3 años. Desde los 25-30 años deben realizarse mamografía, PAP y controles ginecológicos periódicos; en los varones, se realiza control clínico y ecográfico testicular. ${ }^{12,16,18}$

\section{Síndrome de poliposis juvenil (JPS)}

La incidencia es de 1/100 000-160000 individuos. ${ }^{20}$ De herencia autosómica dominante con grado variable de penetrancia, es causado por una mutación germinal inactivadora de los genes SMAD4, BMPR1A y ENG. Estos genes forman parte del factor de crecimiento transformante- $\beta$ (TGF- $\beta$, por su sigla en inglés), un supresor tumoral. Hay historia familiar de pólipos hamartomatosos ("pólipos juveniles") en el 20$50 \%$ de los pacientes. ${ }^{14}$

Desde el punto de vista clínico, puede ser desde asintomático hasta presentarse con obstrucción, invaginación o sangrado gastrointestinal agudo. Las manifestaciones extragastrointestinales incluyen defectos congénitos, como malformaciones de la línea media o cardíacas, anormalidades craneofaciales y polidactilia. ${ }^{20}$

La edad media de presentación de CCR es de 34 años (rango: 15-39 años), y el riesgo acumulativo de CCR es del $38 \%$ a lo largo de la vida. ${ }^{14,16}$

Se recomienda realizar VEDA, VCC desde los 15 años cada 3 años. ${ }^{12,16}$ La cirugía profiláctica se considera en pacientes con pólipos colorrectales con difícil manejo endoscópico (>50-100 pólipos), sangrado grave o diarrea, presencia de pólipos juveniles con displasia, o historia familiar de CCR. ${ }^{12,20}$

\section{E. Síndrome de Lynch (SL) o carcinoma colorrectal hereditario no polipósico (HNPCC)}

De herencia autosómico dominante, se origina por mutaciones monoalélicas en alguno de los 4 genes MMR: $M L H-1$, MSH-2, PMS-2 y MSH-6. La patogénesis de este síndrome se asocia a la acumulación de errores de secuenciación durante la replicación del ADN con la subsiguiente inestabilidad en los MSI.

Los pacientes presentan predisposición al CCR (el $80 \%$ lo desarrollarán a lo largo de su vida) y al carcinoma endometrial $(40 \%)$, entre otros tumores. ${ }^{4}$ Sin embargo, no se asocia a riesgo aumentado de enfermedades malignas en edad pediátrica. $^{21}$

Los criterios diagnósticos clínicos (Criterios de Ámsterdam II) se detallan en la Tabla 1.

Es responsable del 1-5\% de los CCR con una edad media de presentación de 45 años. ${ }^{14}$ En general, se presenta en estadios localizados (el 78-95\% en estadios I y II), el 50-60\% en colon derecho, y tiene un pronóstico más favorable. Presenta mayor incidencia de tumores sincrónicos (CCR dentro de los 6 meses de la cirugía del tumor primario) y metacrónicos (CCR luego de los 6 meses de la cirugía del primario).

Todos los pacientes menores de 45 años con diagnóstico de CCR, según los criterios de Bethesda desarrollados por el National Cáncer Institute, ${ }^{22}$ tienen indicación de búsqueda de MSI.

En los pacientes con SL se recomienda realizar VCC desde los 18-20 años, cada uno o dos años. ${ }^{23,24}$

Cuando la MSI asocia CCR con tumores de SNC, se denomina síndrome de Turcot. ${ }^{7}$ 


\section{F. Síndrome de deficiencia constitucional de MMR (CMMRD)}

Se debe a la mutación germinal bialélica de uno de los cuatro genes $M M R$ y da un fenotipo diferente al SL: alto riesgo de desarrollar enfermedades oncológicas (hematológicas, del SNC e intestinales) en las primeras dos décadas de la vida, múltiples pólipos adenomatosos (1050) y signos de neurofibromatosis de tipo 1, como manchas de color café con leche en la piel. ${ }^{25} \mathrm{La}$ mortalidad es casi del $100 \%$ a los 35 años. ${ }^{26}$

Se recomienda el inicio del cribado con VEDA, VCC anual desde los 3-5 años de vida y, una vez identificados los pólipos, repetir cada 6 meses. La endoscopia alta con videocápsula se puede postergar hasta los 8 años. ${ }^{25,27,28}$ Dentro del tamizaje del resto de las patologías oncológicas, se recomienda realizar ecografía cerebral al nacimiento y luego resonancia magnética nuclear cada 6 meses; laboratorio con hemograma, eritrosedimentación y LDH cada 4 meses; y ecografía ginecológica y urinaria anual en la edad adulta. ${ }^{27}$

Ante el diagnóstico de CMMRD, se debe realizar el tamizaje familiar de mutaciones de los genes MMR. ${ }^{29}$ Los hermanos tienen el $25 \%$ de probabilidades de presentar CMMRD y el $50 \%$, de tener SL.

\section{G. Enfermedad inflamatoria intestinal (EII): colitis ulcerosa y enfermedad de Crohn}

A menor edad de diagnóstico de EII ( $<25$ años), mayor es el riesgo de desarrollar CCR. Los niños con diagnóstico desde hace más de 5 años de colitis ulcerosa, tienen 19 veces mayor riesgo que la población general. ${ }^{30}$

\section{PRESENTACIÓN CLÍNICA Y DIAGNÓSTICO}

El CCR en pediatría tiene características epidemiológicas, presentación clínica e histológica que difieren de la población general (Figura 2). ${ }^{3}$

En los adultos se encuentran identificados los factores predisponentes (edad, abuso de alcohol, tabaquismo, obesidad, dieta hipercalórica, consumo de carnes rojas y sedentarismo), que no aplican en pediatría. ${ }^{31}$

En pediatría, existe una predominancia en el sexo masculino $(62 \%)$, a diferencia de los adultos, donde la distribución por sexo es equivalente. ${ }^{3,32}$

La forma de presentación se relaciona con el sitio del tumor primario. Los tumores que se desarrollan en el ciego y el colon ascendente (colon derecho) suelen ser masas grandes, y se asocian con anemia crónica y síntomas tardíos. Los tumores en el recto y el sigmoides (colon izquierdo) se vinculan a cambios del hábito

FIGURA 2. Histogramas que comparan las frecuencias relativas de niños, adolescentes y adultos según (A) subtipo histológico, (B) sitio tumoral, (C) estadio de enfermedad y (D) grado histológico
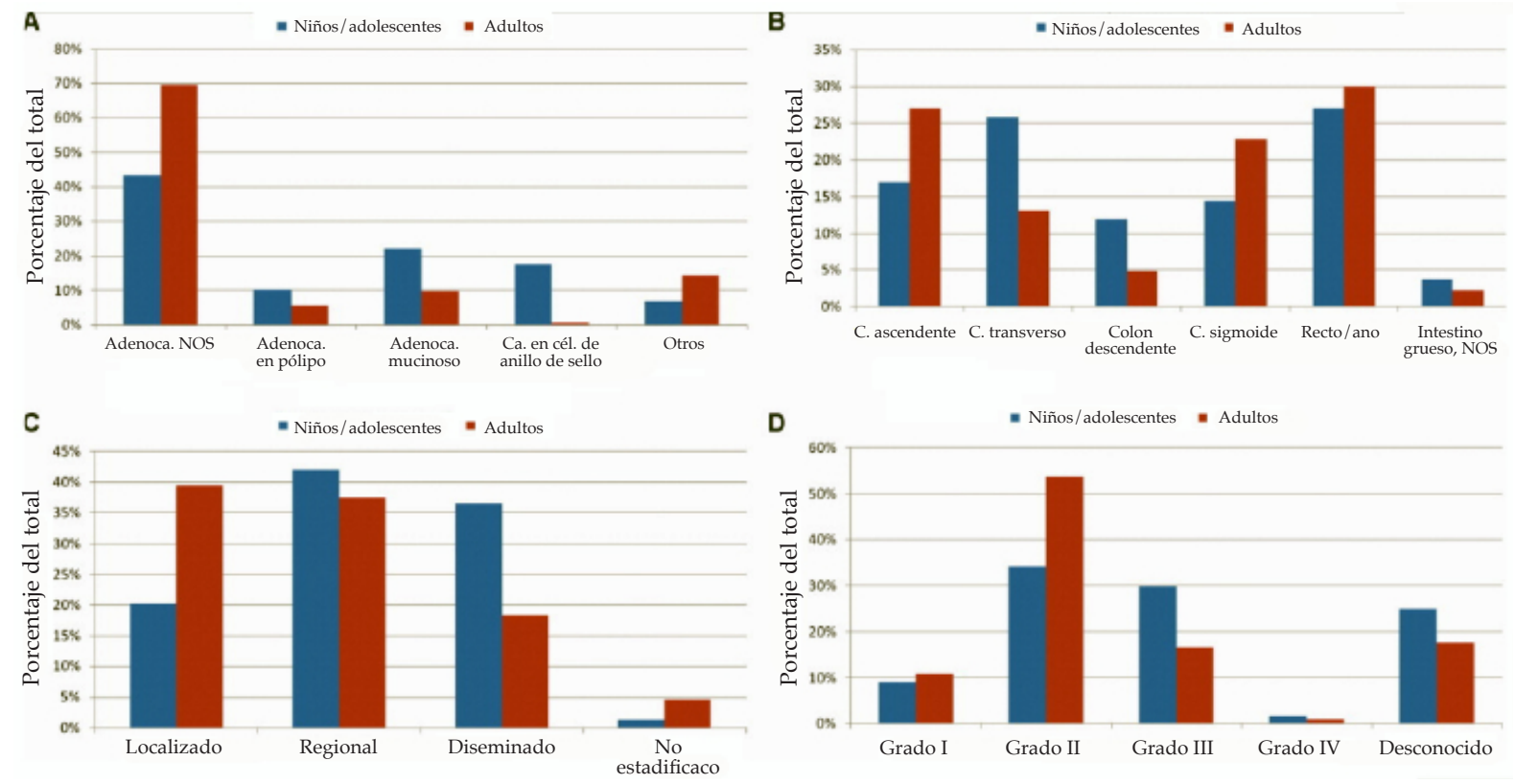

Adenoca: adenocarcinoma. NOS: no clasificado de otra forma. Ca: carcinoma. Cél.: células. C: colon. Adaptado de Sultan I, et al. ${ }^{3}$ 
e492 / Arch Argent Pediatr 2021;119(5):e487-e498 / Actualización

Tabla 2. Estadificación según American Joint Committee on Cancer. 7. ${ }^{\text {ma }}$ ed. (TNM y estadios Dukes) y sobrevida global

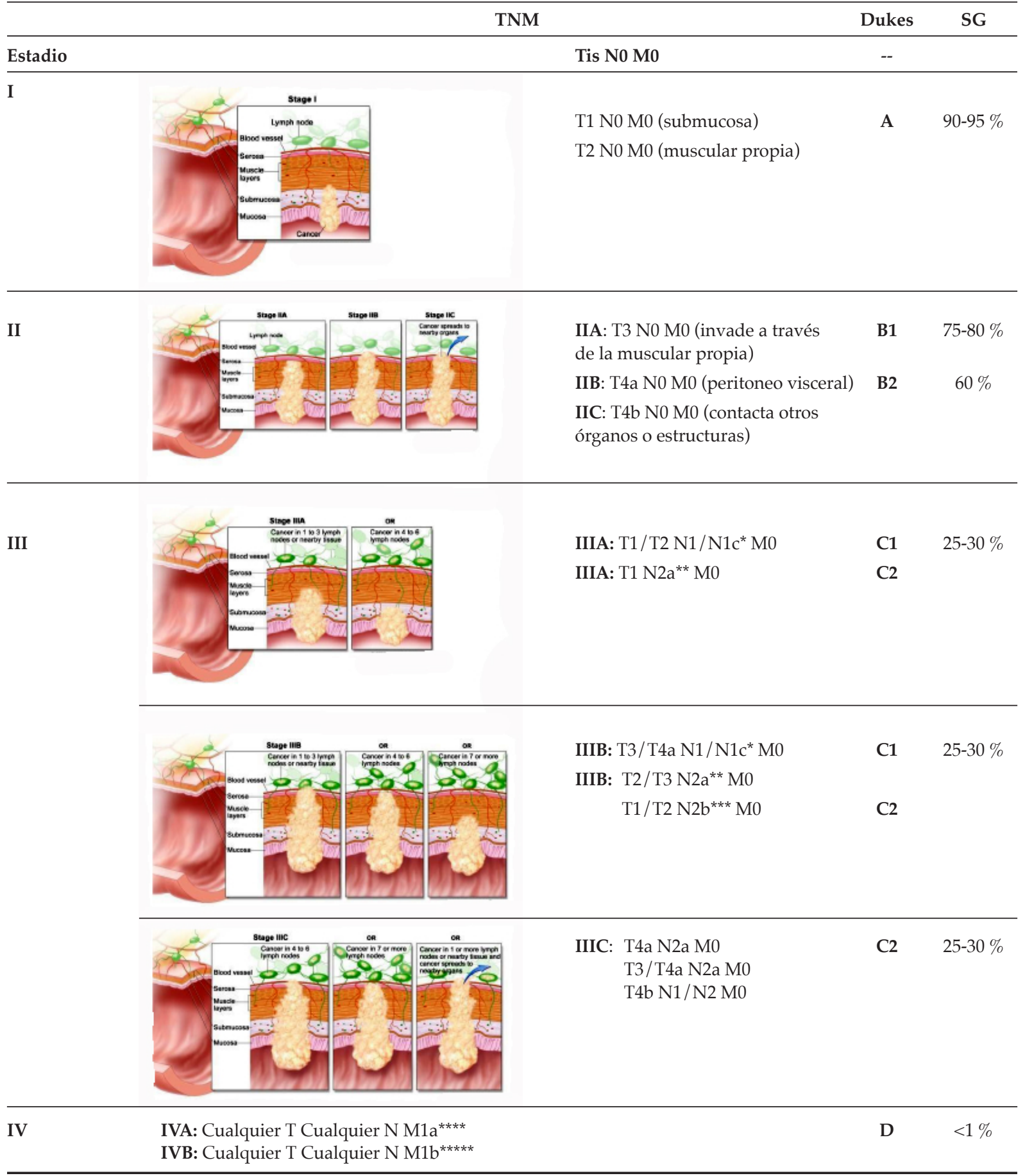

* N1a: un solo ganglio regional, N1b: 2-3 ganglios regionales, N1c: depósitos tumorales en subserosa, mesenterio o tejido pericólico o perirrectal no peritonizado sin compromiso ganglionar regional.

** 4-6 ganglios regionales.

*** 7 o más ganglios regionales.

**** Compromiso confinado a un solo órgano o ganglios a distancia.

***** Compromiso de más de un órgano/sitio o peritoneo.

SG: sobrevida global, AJCC: American Joint Committee on Cancer.

Adaptado de Jessup JM, et al. ${ }^{42}$ 
catártico, disquecia, hematoquecia y anemia. ${ }^{33}$ En pediatría, casi el $80 \%$ se presenta con anemia ferropénica, que es el síntoma más frecuente, seguido por dolor abdominal y pérdida de peso. Un tercio presenta alteración del hábito catártico y hematoquecia. En casos avanzados puede haber obstrucción intestinal y perforación, que se asocian a mal pronóstico. La clínica de abdomen agudo es más común en pediatría $(>20 \%){ }^{1,34}$

Por la infrecuencia de la patología, estos síntomas suelen interpretarse como otras dolencias y el promedio de la demora diagnóstica desde el inicio de los síntomas suele ser de 3 meses, en comparación con un mes para los pacientes mayores de 20 años. ${ }^{1,32,35,36}$ Se ha hipotetizado que dicha demora explicaría, en parte, la presentación en estadios más avanzados. ${ }^{36-38}$

El CCR se clasifica según los siguientes patrones y grados histológicos (frecuencia en pediatría según datos de SEER): ${ }^{3}$

- Patrones histológicos

- Adenocarcinoma no especificado (NOS por sus siglas en inglés) (43\%).

- Adenocarcinoma mucinoso (22\%).

- Carcinoma en células en anillo de sello (18\%).

- Otros (17\%).

- Grado de diferenciación

- Grado I: bien diferenciados (9\%).

- Grado II: moderadamente diferenciados $(34 \%)$.
- Grado III: pobremente diferenciados $(30 \%)$.

- Grado IV: indiferenciados; anaplásicos $(2 \%)$.

- Desconocido (25\%).

Las lesiones de bajo grado (I-II) que no tienen invasión angiolinfática y con márgenes de resección adecuados son clasificadas histológicamente como favorables. ${ }^{35}$

Los pacientes pediátricos tienen mayor incidencia de patrón mucinoso y en células en anillo de sello, además de pobremente diferenciados o indiferenciados., ${ }^{3,34}$ En pediatría, dada la rareza de la patología, la histología no ha demostrado valor pronóstico. ${ }^{35}$

\section{ESTADIFICACIÓN}

Solo el $19 \%$ de los niños y adolescentes presenta enfermedad localizada, con el doble de posibilidades de metástasis a distancia respecto a los adultos. ${ }^{1,3}$ Esta característica limita la posibilidad quirúrgica, principal herramienta curativa.

Las guías de Tumores raros en la edad pediátrica (TREP, por su sigla en italiano) recomiendan la realización de ecografía abdominal, VEDA y VCC como estudios iniciales. Los pacientes con síndromes genéticos predisponentes deben realizarse, además, el estudio con cápsula endoscópica, según indicaciones. ${ }^{39}$

FIGURA 3. Distribución de sobrevida global a 5 años de 11 pacientes con presencia de síndromes genéticos predisponentes (100\%) y de 15 pacientes sin factores genéticos predisponentes $(36,5 \%)(p<0,001)$

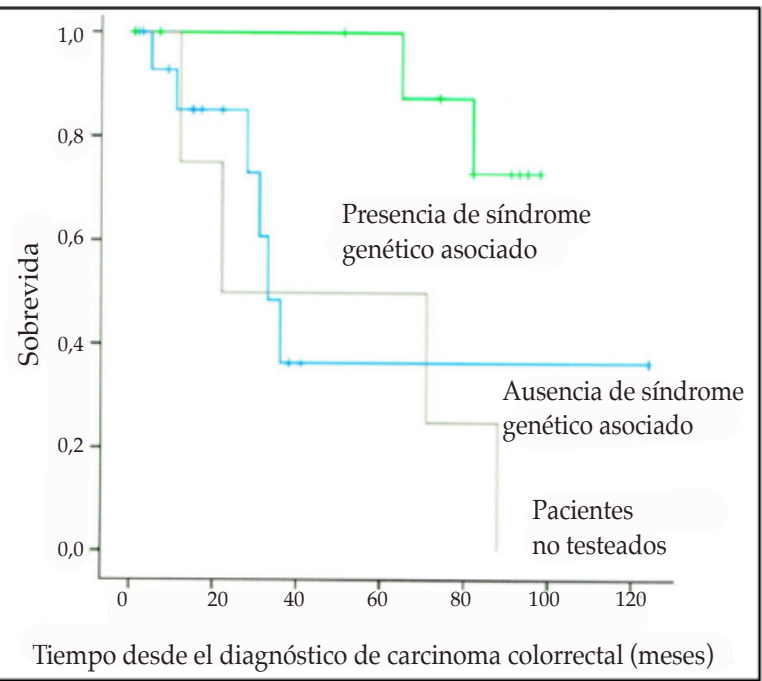


Como estudios de estadificación inicial, se recomiendan tomografía computada (TC) de tórax, abdomen y pelvis, y centellograma con Tc99. Las guías TREP recomiendan la utilización de la TC con emisión de positrones. . $13,39,40^{2}$

La medición de marcadores tumorales (antígeno carcinoembrionario [CEA, por su sigla en inglés] y antígeno carbohidratado 19.9 [CA 19.9, por su sigla en inglés]) puede ser útiles para evaluar la respuesta al tratamiento en pacientes metastásicos y para el monitoreo de recaída. ${ }^{41}$

La guía de la American Joint Committee on Cancer (AJCC, $7 .^{\mathrm{ma}}$ edición) provee el sistema más utilizado de estadificación del CCR combinando el sistema TNM con los estadios pronósticos de Dukes (Tabla 2). ${ }^{42}$ En pediatría, el 18,4 \% de los pacientes se presentan como estadio de Dukes B; el 55,3\% como Dukes C y el 26,3\% como Dukes D; ninguno como Dukes A. ${ }^{1}$

En los pacientes jóvenes, los sitios metastásicos más frecuentes son el peritoneo (34\%), el hígado $(32 \%)$, los pulmones $(9 \%)$, los ovarios $(7 \%)$ y los huesos $(7 \%)$. Este patrón difiere del que se presenta en adultos, en quienes los sitios más habituales de metástasis son el hígado (30-70\%), los pulmones $(20-40 \%)$ y los huesos $(5-10 \%) .1,32$

TABLA 3. Resumen de recomendaciones terapéuticas

\begin{tabular}{|c|c|}
\hline Estadio TNM & Estándar de tratamiento \\
\hline $\begin{array}{l}\text { I } \\
\text { SG } 90 \%\end{array}$ & - Cirugía amplia con anastomosis \\
\hline $\begin{array}{l}\text { II } \\
\text { SG } 72-80 \%\end{array}$ & $\begin{array}{l}\text { - Cirugía amplia con anastomosis } \\
\text { - Quimioterapia adyuvante controversial (basada en 5-FU). } \\
\text { - Considerar como factores de riesgo de recurrencia: muestreo ganglionar inadecuado (<12 ganglios), } \\
\text { T4-T3, perforación u oclusión, compromiso de peritoneo visceral, márgenes de resección } \\
\text { inadecuados, histología pobremente diferenciada. } \\
\text { - Oxaliplatino + fluoropirimidinas son tratamiento estándar. }\end{array}$ \\
\hline $\begin{array}{l}\text { III } \\
\text { SG } \\
\text { IIIA } 60 \%\end{array}$ & $\begin{array}{l}\text { - Cirugía amplia con anastomosis } \\
\text { - Quimioterapia neoadyuvante }\end{array}$ \\
\hline $\begin{array}{l}\text { IIIB } 42 \% \\
\text { IIIC } 27 \%\end{array}$ & $\begin{array}{l}\text { - Oxaliplatino + fluoropirimidinas son el estándar de tratamiento, con preferencia por FOLFOX } \\
\text { o regímenes con capecitabina oxaliplatino. } \\
\text { Pacientes con }>3 \text { nódulos tienen peor pronóstico. }\end{array}$ \\
\hline IV & $\begin{array}{l}\text { Tratamiento quirúrgico es lo único potencialmente curativo. Se indica para: } \\
\text { - Resección con anastomosis del primario con intención curativa. } \\
\text { - Bypass de zona obstructiva o zona sangrante en casos seleccionados con intenciones paliativas. } \\
\text { - Resección en casos seleccionados de lesiones metastásicas hepáticas, pulmonares solitarias u } \\
\text { ováricas con intención curativa. } \\
\text { - Radioterapia paliativa } \\
\text { - Quimioterapia paliativa: } 1 \text { ra }^{\text {ra }} \text { línea con FOLFOX, FOLFIRI, FOLFOXIRI CAPOX o FUOX, con o sin } \\
\text { bevacizumab (no tienen un papel claro).47,48,50 } \\
\text { 4. Quimioterapia dirigida para pacientes en los que fracasa la primera línea de tratamiento: } \\
\text { - Cetuximab: no sirve para pacientes con KRAS mutado. } \\
\text { - Ziv-aflibercept: anti-VEGF. } \\
\text { - Ramucirumab: anticuerpo monoclonal humanizado que se une al VEGF-2. } \\
\text { - Panitumumab: anticuerpo humanizado contra EGFR en pacientes KRAS-wild type. } \\
\text { - Regorafenib: inhibidor de múltiples tirosina-cinasas, entre las cuales VEGF. } \\
\text { - Trifluridina y tipiracil (TAS-102): análogo de timidina. } \\
\text { - Pembrolizumab: anticuerpo PD-1 utilizado para pacientes con fenotipo de inestabilidad de } \\
\text { microsatélites (MSI-H) (4 \% de los pacientes en estadio IV). }{ }^{48,50,51}\end{array}$ \\
\hline
\end{tabular}

SG: sobrevida global, 5-FU: fluorouracilo, MSI: inestabilidad microsatétite (por su sigla en inglés),

FOLFOX: leucovorina + fluorouracilo + oxaliplatino, FOLFIRI: leucovorina + fluorouracilo + irinotecán,

FOLFOXIRI: leucovorina + fluorouracilo + oxaliplatino + irinotecán, CAPOX: capecitabina + oxaliplatino,

FUOX: fluorouracilo + oxaliplatino, anti-VEGF: anti-factor de crecimiento endotelial vascular (por su sigla en inglés),

EGFR: receptor del factor de crecimiento epidérmico (por su sigla en inglés). 


\section{FACTORES PRONÓSTICOS Y SOBREVIDA}

Los pacientes con HNPCC tienen mejor pronóstico que aquellos con enfermedad esporádica, independientemente de la estadificación inicial del tumor. ${ }^{5}$ Mork et al., ${ }^{43}$ identificaron, en una cohorte de 193 pacientes menores de 35 años, un $35 \%$ de pacientes con síndromes hereditarios predisponentes: los pacientes sin síndromes hereditarios eran más propensos a tener tumores del lado izquierdo, enfermedad metastásica e histología desfavorable. Weber et al. ${ }^{44}$ también evidenciaron que los pacientes con síndromes de predisposición (principalmente HNPCC) mostraron una patología menos agresiva con mejor sobrevida (Figura 3).

Los factores pronósticos desfavorables para la población pediátrica son el grado histológico IIIIV, la histología mucinosa o en célula en anillo de sello, y el estadio inicial avanzado con invasión

Figura 4. Curvas de estimación de sobrevida de Kaplan-Mayer para niños y adolescentes comparadas según (A) grado tumoral, (B) subtipo histológico, y (C) estadio de la enfermedad
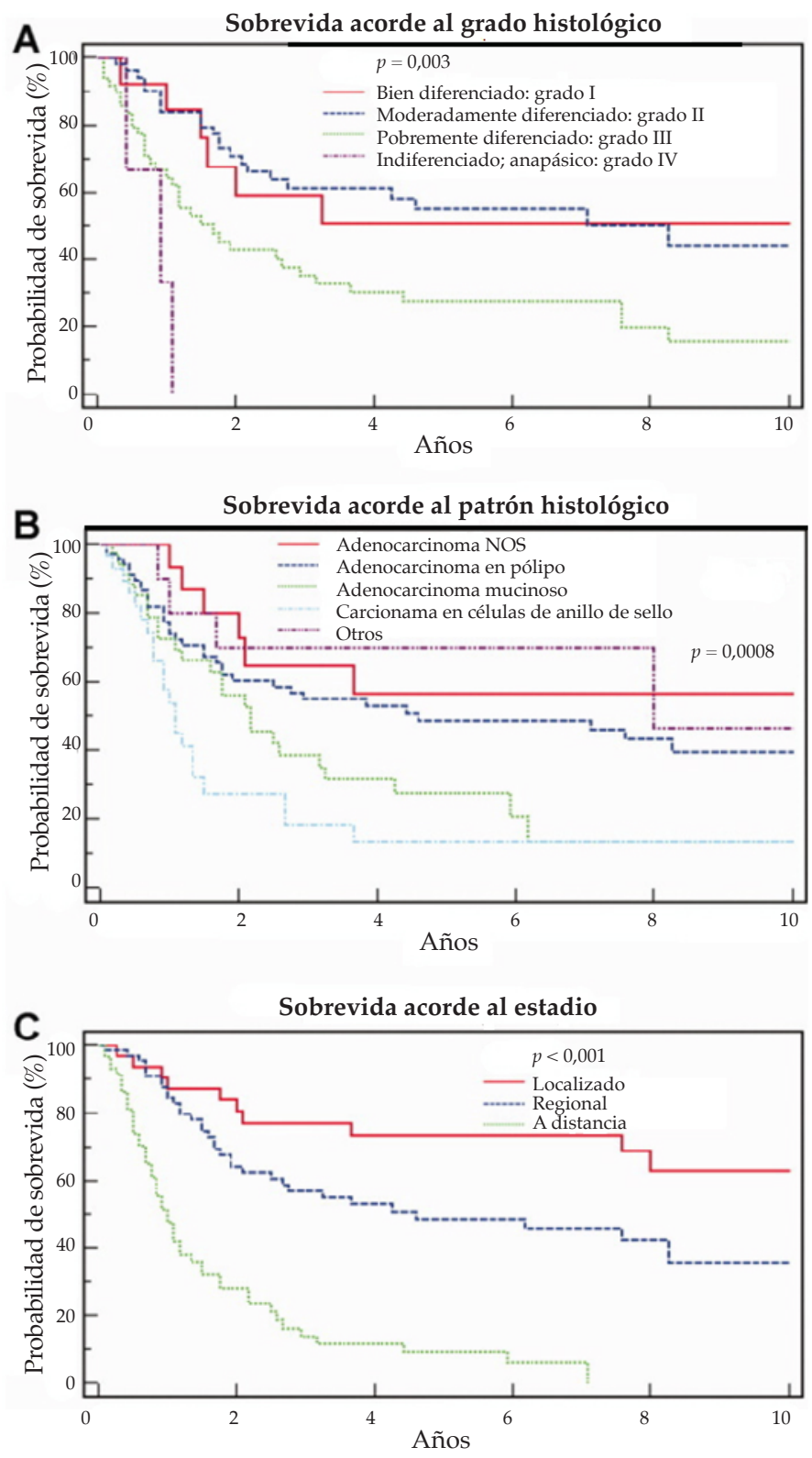

NOS: no especificado de otra forma. 
regional y / o a distancia. En quienes se logra la resección completa, la identificación de invasión tumoral de la serosa y la presencia de ganglios positivos son factores independientes de mal pronóstico (Figura 4). ${ }^{1,3}$

En pediatría, la SG estimada a 5 y 10 años según los datos de SEER es del $40 \%(4,2)$ y del $31 \%(4,4)$, respectivamente. ${ }^{2,3}$ La sobrevida libre de evento (SLE) a 10 años es del 17,7 \% (5,1). ${ }^{31}$ Estos valores difieren de la población adulta, cuya SG a 5 y 10 años es de $60 \%(0,1)$ y $54 \%(0,1)$ respectivamente $(p<0,001)$ (Figura 5). ${ }^{3}$

La SG a 5 años en estadio M1a es del 20,6\% versus el 7,7 \% en los pacientes M1b..$^{32,41}$

\section{TRATAMIENTO}

Las recomendaciones para pediatría son adaptaciones de la experiencia en adultos. ${ }^{39}$

\section{- Cirugía}

La cirugía es el pilar del tratamiento y debe ser radical. Sin la resección quirúrgica completa, la curación no es posible. ${ }^{39}$ Incluso puede ser curativa en pacientes con metástasis pulmonares o hepáticas resecables. Es necesario resecar márgenes $\geq 5 \mathrm{~cm}$ de intestino normal para evitar recurrencias en la zona de la anastomosis. Se debe examinar al menos 12 ganglios negativos para definir la enfermedad como estadio N0. ${ }^{31}$ Se debe explorar la superficie peritoneal incluida la fascia renal y el diafragma. Todos los ganglios peritoneales deberían ser resecados.

En pediatría, raramente el CCR es el diagnóstico de sospecha inicial. En caso de cirugía oncológicamente inadecuada, se indica la reexploración quirúrgica para asegurar los márgenes y el examen linfático necesario.

\section{- Quimioterapia}

La indicación del tratamiento adyuvante depende del estadio inicial y se siguen las guías para pacientes adultos. ${ }^{39}$

Los pacientes en estadio I tienen una sobrevida del $90 \%$ a 5 años solo con cirugía y se recomienda observación cercana, sin requerimiento de quimioterapia adyuvante.

En estadio II, el papel de la quimioterapia adyuvante no es claro, y no parecería mejorar la SG en más del $5 \%$. La mayoría de los pacientes pediátricos y adolescentes con estadio II presentan factores pronósticos desfavorables, por lo que debe considerarse la quimioterapia adyuvante. ${ }^{31,45}$

En estadios III-IV (compromiso ganglionar y/o metastásico) la quimioterapia tiene un claro beneficio. ${ }^{39} \mathrm{El}$ manejo es controversial. Se realiza quimioterapia basada en 5-fluorouracilo y

Figura 5. Gráficos de sobrevida estimada comparando ( $A$ ) niños, adolescentes y adultos ( $p<0,001, \log$-rank test), (B) solo niños $y$ adolescentes, $y(C)$ solo adultos durante 3 períodos

A

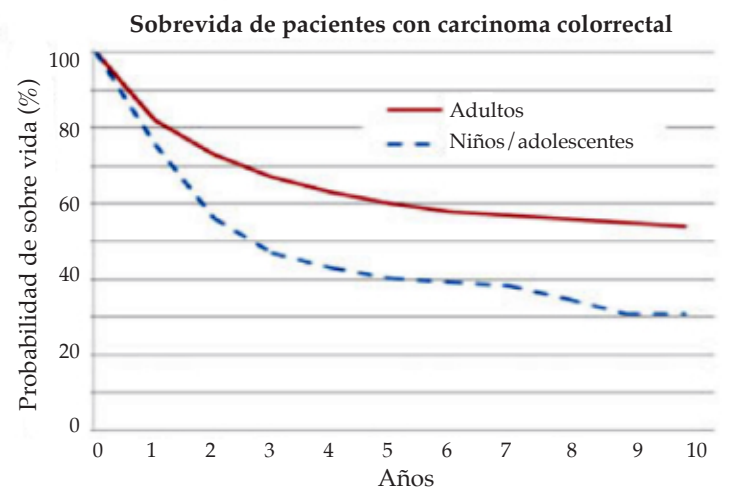

$\mathbf{B}$

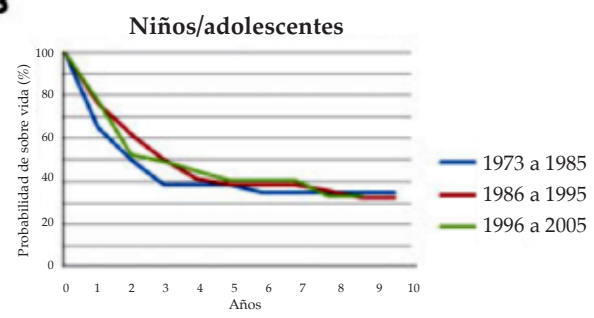

Adaptada de Sultan I, et al. ${ }^{3}$
C

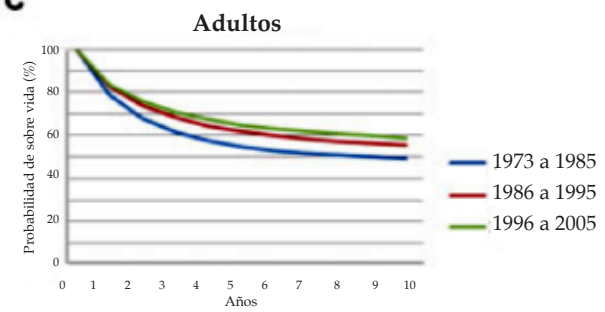


ácido folínico (5-FU-LV), asociando agentes que demostraron utilidad: capecitabina, oxaliplatino e irinotecán. ${ }^{31}$

\section{- Terapias dirigidas}

La terapia dirigida ha demostrado ser beneficiosa para algunos pacientes, principalmente para los estadios avanzados (IIIIV). Los agentes terapeúticos más estudiados en CCR son el bevacizumab, el pembrolizumab, el cetuximab, el panitumumab, el bortezomib y el gefitinib. ${ }^{31,46-51}$

\section{- Radioterapia}

El papel de la radioterapia se limita al uso posquirúrgico en pacientes con cáncer rectal, combinada con quimioterapia a base de 5-FU. ${ }^{39}$ En casos avanzados (estadio T4, perforación local u obstrucción) se puede utilizar en forma preoperatoria para disminuir las probabilidades de recurrencias posquirúrgicas o para permitir procedimientos quirúrgicos menos invasivos.

En la Tabla 3 se resumen las recomendaciones terapéuticas.

\section{CONCLUSIÓN}

En pediatría, el CCR presenta características biológicas propias, que difieren del adulto, y constituye una subentidad específica de tumores raros en niños.

Dada la baja incidencia del CCR en este grupo etario, consideramos fundamental la realización de un trabajo en red con centros especializados de adultos, a fin de lograr un abordaje integral, con tratamientos adecuados en tiempo y forma, bajo la guía de expertos en esta patología.

En el CCR se encuentra una clara asociación con síndromes de predisposición genética familiar al cáncer con manifestaciones que, si bien en su mayoría se presentan en edad adulta, la pesquisa precoz con tamizaje adecuado permite prevenir y tratar oportunamente. A su vez, es importante para el pediatra poder brindar un adecuado consejo genético para el grupo familiar del paciente.

\section{REFERENCIAS}

1. Kaplan M, Isikdogan A, Gumus M, Arslan U, et al. Childhood, adolescents, and young adults $(\leq 25 \mathrm{y})$ colorectal cancer: study of Anatolian Society of Medical Oncology. J Pediatr Hematol Oncol. 2013; 35(2):83-9.

2. Brench I, Kaatsch P. Epidemiology. In Schneider D, Brecht I, Olson T, Ferrari A (eds). Rare tumors in children and adolescents. Berlin, Heidelberg: Springer-Verlag; 2012. Págs.43-61.
3. Sultan I, Rodriguez-Galindo C, El-Taani H, Pastore G, et al. Distinct features of colorectal cancer in children and adolescents: a population-based study of 159 cases. Cancer. 2010; 116(3):758-65.

4. Olson T, Schneider D, Brecht I, Ferrari A. Rare Tumors: A Different Perspective on Oncology. In: Schneider D, Brecht I, Olson T, Ferrari A (eds). Rare tumors in children and adolescents. Berlin, Heidelberg:Springer-Verlag; 2012. Págs.3-13.

5. Gryfe R, Kim H, Hsieh E, Aronson M, et al. Tumor microsatellite instability and clinical outcome in young patients with colorectal cancer. NEnglJMed.2000;342(2):6977.

6. Tariq K, Ghias K. Colorectal cancer carcinogenesis: a review of mechanisms. Cancer Biol Med. 2016; 13(1):120-35.

7. SingerG,Hoellwarth ME. Colorectal carcinomas in children: an institutional experience. Pediatr Surg Int. 2012;28(6):5915.

8. Lynch HT, de la Chapelle A. Hereditary colorectal cancer. N Engl J Med. 2003; 348(10):919-32.

9. Vasen HF, Möslein G, Alonso A, Aretz S, et al. Guidelines for the clinical management of familial adenomatous polyposis (FAP). Gut. 2008; 57(5):704-13.

10. Septer S, Lawson CE, Anant S, Attard T. Familial adenomatous polyposis in pediatrics: natural history, emerging surveillance and management protocols, chemopreventive strategies, and areas of ongoing debate. Fam Cancer. 2016; 15(3):477-85.

11. Hyer W, Cohen S, Attard T, Vila-Miravet V, et al. Management of Familial Adenomatous Polyposis in Children and Adolescents: Position Paper From the ESPGHANPolyposis Working Group.JPediatr Gastroenterol Nutr. 2019; 68(3):428-41.

12. Achatz MI, Porter CC, Brugières L, Druker H, et al. Cancer Screening Recommendations and Clinical Management of Inherited Gastrointestinal CancerSyndromes in Childhood. Clin Cancer Res. 2017; 23(13):e107-14.

13. Möslein G, PistoriusS, Saeger HD, SchackertHK. Preventive surgery for colon cancer in familial adenomatous polyposis and hereditary nonpolyposis colorectal cancer syndrome. Langenbecks Arch Surg. 2003; 388(1):9-16.

14. Bertario L, Signoroni S. Gastrointestinal Cancer Predisposition Syndromes. In: Schneider DT, Brecht IB, Olson TA, Ferrari A (eds). Rare tumors in children and adolescents. Berlin Heidelberg: Springer-Verlag; 2012. Págs.255-62.

15. Vitellaro M, Ferrari A, Trencheva K, Sala P, et al. Is laparoscopic surgery an option to support prophylactic colectomy in adolescent patients with Familial Adenomatous Polyposis (FAP)? Pediatr Blood Cancer. 2012; 59(7):1223-8.

16. Guillén-Ponce $C$, Serrano R, Sánchez-Heras AB, Teulé A, et al. Clinical guideline seom: hereditary colorectal cancer. Clin Transl Oncol. 2015; 17(12):962-71.

17. Nieuwenhuis $M H$, Vogt $S$, Jones $N$, Nielsen $M$, et al. Evidence for accelerated colorectal adenoma-carcinoma progression in MUTYH-associated polyposis? Gut. 2012; 61(5):734-8

18. Latchford A, CohenS, AuthM,ScaillonM, etal.Management of Peutz-Jeghers Syndrome in Children and Adolescents: A Position Paper From the ESPGHAN Polyposis Working Group. J Pediatr Gastroenterol Nutr. 2019; 68(3):442-52.

19. Giardiello FM, Trimbath JD. Peutz-Jeghers syndrome and management recommendations. Clin Gastroenterol Hepatol. 2006; 4(4):408-15.

20. IshidaH, Ishibashi K, Iwama T. Malignanttumors associated with juvenile polyposis syndrome in Japan. Surg Today. 2018; 48(3):253-63. 
21. HeathJA, ReeceJC, BuchananDD, Casey G, etal. Childhood cancers in families with and without Lynch syndrome. Fam Cancer. 2015; 14(4):545-51.

22. Umar A, Boland R, Terdiman J, Syngal S, et al. Revised Bethesda Guidelines for hereditary nonpolyposis colorectal cancer (Lynch syndrome) and microsatellite instability. J Natl Cancer Inst. 2004; 96(4):261-8.

23. Vasen H, Blanco I, Aktan-Collan K, Gopie JP, et al. Revised guidelines for the clinical management of Lynch syndrome (HNPCC) recommendations by a group of European experts. Gut. 2013; 62(6):812-23.

24. Seppälä TT, Pylvänäinen K, Mecklin JP. Uptake of genetic testing by the children of Lynch syndrome variant carriers across three generations. Eur J Hum Genet. 2017; 25(11):123745.

25. Aronson M, Gallinger S, Cohen Z, Cohen S, et al. Gastrointestinal Findings in the Largest Series of Patients With Hereditary Biallelic Mismatch Repair Deficiency Syndrome: Report from the International Consortium. Am J Gastroenterol. 2016; 111(2):275-84.

26. Antelo M, Milito D, Rhees J, Roca E, et al. Pitfalls in the diagnosis of biallelic PMS2 mutations. Fam Cancer. 2015; 14(3):411-4.

27. Durno C, Aronson M, Tabori U, Malkin D, et al. Oncologic surveillance for subjects with biallelic mismatch repair gene mutations: 10 year follow-up of a kindred. Pediatr Blood Cancer. 2012; 59(4):652-6.

28. Levi Z, Kariv R, Barnes-Kedar I, Goldberg Y, et al. The gastrointestinal manifestation of constitutional mismatch repair deficiency syndrome: from a single adenoma to polyposis-like phenotype and early onset cancer. Clin Genet. 2015; 88(5):474-8.

29. Westdorp H, Kolders S, Hoogerbrugge N, de Vries IJ, et al. Immunotherapy holds the key to cancer treatment and prevention in constitutional mismatch repair deficiency (CMMRD) syndrome. Cancer Lett. 2017; 403:159-64.

30. Peneau A, Savoye G, Turck D, Dauchet L, et al. Mortality and cancer in pediatric-onset inflammatory bowel disease: a population-based study. Am J Gastroenterol. 2013; 108(10):1647-53.

31. GoldbergJ, FurmanW. Management of colorectal carcinoma in children and young adults. J Pediatr Hematol Oncol. 2012; 34(Suppl 2):S76-9.

32. Hill D, Furman W, Billups C, Riedley S, et al. Colorectal carcinoma in childhood and adolescence: a clinicopathologic review. J Clin Oncol. 2007; 25(36):5808-14.

33. Al-Tonbary Y, Darwish A, El-Hussein A, Fouda A. Adenocarcinoma of the colon in children: case series and mini-review of the literature. Hematol Oncol Stem Cell Ther. 2013; 6(1):29-33.

34. Kaplan MA, Ozaydin S, Yerlikaya H, Karaagac M, et al. Clinicopathologic and prognostic differences between three different age groups (child/adolescent, young adults, and adults) of colorectal cancer patients: a multicentre study. Oncol Res Treat. 2019; 42(10):516-22.

35. Shehata B, ShulmanS. Pathology and Differential Diagnosis. In: Schneider DT, Brecht IB, Olson TA, Ferrari A (eds). Rare tumors in children and adolescents. Berlin, Heidelberg: Springer-Verlag 2012.Págs.245-54.
36. Chantada G, Perelli V, García Lombardi M, AmaralD, et al. Colorectal carcinoma in children, adolescents, and young adults. J Pediatr Hematol Oncol. 2005; 27(1):39-41.

37. KarnakI, Ciftci A, SenocakM, Büyükpamukçu N. Colorectal carcinoma in children. J Pediatr Surg. 1999;34(10):1499-504.

38. Lamego C, Torloni H. Colorectal adenocarcinoma in childhood and adolescent. Report of 11 cases and review of the literature. Pediatr Radiol. 1989; 19(8):504-8.

39. Indini A, Bisogno G, Cecchetto G, Vitellaro M, et al. Gastrointestinal tract carcinoma in pediatric and adolescent age: the TREP project experience. Pediatr Blood Cancer. 2017; 64(12):1-8.

40. Kunawudhi A, SereeborwornthanasakK, Promteangtrong C, Siripongpreeda B, et al. Value of FDG PET/ContrastEnhanced CT in Initial Staging of Colorectal Cancer Comparison with Contrast-Enhanced CT. Asian Pac JCancer Prev. 2016; 17(8):4071-5.

41. Ferrari A, Casanova M, Massimino M, Sultan I. Peculiar features and tailored management of adult cancers occurring in pediatric age. Expert Rev Anticancer Ther. 2010; 10(11):1837-51.

42. Jessup JM, Goldberg RM, Asare EA, et al. Colon and rectum. In: Amin MB, Edge SB, Greene FL, Byrd F, et al. (eds). AJCC Cancer Staging Manual. 8th ed. New York, NY: Springer, 2017.Págs.251-74.

43. Mork M, You Y, Ying J, Bannon S, et al. High prevalence of hereditary cancer syndromes in adolescents and young adults with colorectal cancer. JClin Oncol. 2015;33(31):35449.

44. Weber M, Schneider D, Offenmüller S, Kaatsch P, et al. Pediatric Colorectal Carcinoma is Associated With Excellent Outcome in the Context of Cancer Predisposition Syndromes. Pediatr Blood Cancer. 2016; 63(4):611-7.

45. Zhou Z, Wu X, Wang R, Li L, et al. Optimal use of adjuvant chemotherapy in stage II colorectal cancer. Int J Colorectal Dis. 2011; 26(7):867-73.

46. Hurwitz H, Fehrenbacher L, Novotny W, Cartwright $\mathrm{T}$, et al. Bevacizumab plus irinotecan, fluorouracil, and leucovorin for metastatic colorectal cancer. $N$ Engl J Med. 2004; 350(23):2335-42.

47. Saltz L, Clarke S, Díaz-Rubio E, Scheithauer W, et al. Bevacizumab in combination with oxaliplatin-based chemotherapy as first-line therapy in metastatic colorectal cancer: a randomized phase III study. J Clin Oncol. 2008; 26(12):2013-9.

48. Marcus L, Lemery SJ, Keegan P, Pazdur R. FDA Approval Summary: Pembrolizumab for the Treatment of Microsatellite Instability-High Solid Tumors. Clin Cancer Res. 2019; 25(13):3753-8.

49. Saltz L. Adjuvant therapy for colon cancer. Surg Oncol Clin N Am. 2010; 19(4):819-27.

50. Salman P, Panay S, Fernández R, Mahave M, Soza-Ried C. Evidence of response to pembrolizumab in a patient with Lynch syndrome-related metastatic colon cancer. Onco Targets Ther. 2018; 11:7295-300.

51. Benson A 3rd, Venook A, Cederquist L, Chan E, et al. Colon Cancer, Version 1.2017, NCCN Clinical Practice Guidelines in Oncology. J Natl Compr Canc Netw. 2017; 15(3):370-98. 